\title{
DESCRIÇÃO EM BIBLIOTECAS, ARQUIVOS, MUSEUS E GALERIAS DE ARTE: LINKANDO RECURSOS E COMUNIDADES
}

\section{DESCRIPCIÓN EN BIBLIOTECAS, ARCHIVOS, MUSEOS Y GALERIAS DE ARTE: LINKANDO RECURSOS Y COMUNIDADES}

\author{
Ana Carolina Simionato* \\ Felipe Augusto Arakaki** \\ Plácida Leopoldina Ventura Amorim da Costa Santos ${ }^{* * *}$
}

\begin{abstract}
RESUMO:
Introdução: A plataforma Web e as tecnologias possibilitaram diferentes formas de interação entre bibliotecas, arquivos, museus, galerias de arte e seus respectivos públicos. A constante necessidade de atualização do profissional da informação diante o desenvolvimento tecnológico, contextualizam a problemática desse estudo, que consiste em identificar quais as tendências para otimizar os canais de comunicação entre usuário e os recursos informacionais, ampliando as possibilidades de representação em arquivos, bibliotecas, museus e galerias de arte. Objetivo: $O$ objetivo é apresentar as temáticas emergentes no âmbito da representação da informação na plataforma Web para profissionais da informação no contexto de bibliotecas, arquivos, museus e galerias de arte. Metodologia: Caracteriza-se como um estudo teórico, pois busca discutir sob a óptica da representação da informação os desafios para integração e cooperação de diferentes contextos, unificados pelas descrições de recursos informacionais, possibilitando assim, a navegação do usuário nos ambientes informacionais e a localização de outras informações e recursos informacionais. Resultados: Diante desse contexto, foi levantado os princípios de representação em bibliotecas, arquivos, museus e galerias de arte, buscando evidenciar suas características e percepções das necessidades dos usuários. Posteriormente foi discutido as tendências e desafios para os profissionais da informação no intuito de reduzir os esforços da representação para criar possibilidades de navegação aos usuários. Conclusões: Por fim, foram apresentadas algumas iniciativas que tem o propósito de criar sistemas que são capazes de ligar dados de ambientes informacionais distintos. Conclui-se que a proposta do reaproveitamento

*Doutora Ciência da Informação Unesp. Docente do Departamento de Ciência da Informação, da Universidade Federal de São Carlo - UFSCar. E-mail: simionato.ac@gmail.com

**Doutorando em Ciência da Informação pela Universidade Estadual Paulista - UNESP. E-mail: fe.arakaki@gmail.com.

***Doutora em Semiótica e Linguistica Geral pela FFLCH/USP. Docente do Programa de PósGraduação em Ciência da Informação da FFC/UNESP. E-mail: placida@marilia.unesp.br.
\end{abstract}

Inf. Inf., Londrina, v. 22, n. 2, p. 449 - 466, maio/ago., 2017. 
Ana Carolina Simionato, Felipe Augusto Arakaki, Plácida Leopoldina Ventura Amorim da Costa Santos

Descrição em bibliotecas, arquivos, museus e galerias de arte: linkando recursos e comunidades

dos dados parece ser uma alternativa para minimizar esforços do retrabalho do profissional.

Palavras-chave: Bibliotecas, arquivos, museus e galerias de arte. Web Semântica. Linked Data. Descrição e catalogação.

\section{INTRODUÇÃO}

A temática do VII Seminário em Ciência da Informação (SECIN), no ano de 2017, "Linkando informações, culturas e comunidades: reflexões teóricopráticas" faz refletirmos sobre as tendências da descrição de recursos digitais no ambiente Web, principalmente as possibilidades da aplicação dos princípios do Linked Data. Essas tecnologias possibilitaram diferentes formas de integração entre bibliotecas, arquivos, museus, galerias de arte e seus respectivos públicos.

Nesse cenário, os diversos tipos de catálogos são considerados como uma forma de mediação entre os recursos informacionais e seus usuários. 0 recurso informacional é a "[...] informação objetivada no contexto de um campo do conhecimento podendo ser apresentado em uma estrutura analógica e/ou digital [...]" (SANTOS; SIMIONATO; ARAKAKI, 2014, p.148).

Para que os catálogos sistematizem melhores possibilidades de acesso, de busca e de recuperação aos recursos informacionais, são delineadas colaborações entre diversas áreas para garantir a comunicação entre os sistemas representacionais e organizacionais em comum acordo com as necessidades do usuário. Ou seja, um dos campos de estudo da Ciência da Informação que de acordo com Santos e Sant'Ana (2013, p.200) pode ser considerada como uma área do conhecimento focada nas "[...] metodologias e nos instrumentos desenvolvidos ao longo do tempo para armazenar, descrever, recuperar, preservar, disseminar e compartilhar as experiências humanas."

Nesse contexto, a proposta desse estudo é identificar quais as tendências para otimização dos canais de comunicação entre usuário e os 
Ana Carolina Simionato, Felipe Augusto Arakaki, Plácida Leopoldina Ventura Amorim da Costa Santos

Descrição em bibliotecas, arquivos, museus e galerias de arte: linkando recursos e comunidades

recursos informacionais, na perspectiva de ampliar as possibilidades de representação de recursos em arquivos, bibliotecas, museus e galerias de arte.

O objetivo do trabalho é apresentar as temáticas emergentes no âmbito da representação de recursos na plataforma Web para profissionais da informação no contexto das instituições de informação. Sendo caracterizado como um estudo teórico, pois busca discutir sob a óptica da representação, os desafios para integração e cooperação de diferentes contextos, unificados pelas descrições de recursos informacionais na plataforma Web, possibilitando assim, a navegação do usuário nos ambientes informacionais e a busca e a localização de informações e recursos informacionais.

Foi utilizado também, o método perspectivismo proposto por Peterson (1996) na abordagem do universo metodológico da Ciência da Informação para a "[...] análise dos processos de adequação e ajuste de uma perspectiva adotada por um sujeito em seu contexto específico." (SANTOS; VIDOTTI, 2009, não paginado). Na qual a percepção do usuário pode variar dependendo do contexto em que ele está (biblioteca, arquivo, museus ou galerias de arte). Um mesmo recurso informacional pode pertencer a diferentes contextos, sua representação básica poderá ser a mesma, entretanto, as descrições adicionais, caracterizarão as exigências dos diferentes contextos, no atendimento das necessidades informacionais dos usuários.

Identificados os princípios de representação em bibliotecas, arquivos, museus e galerias de arte, buscando evidenciar suas características e percepções sobre as necessidades dos usuários, foram discutidos as tendências e os desafios, no intuito de reduzir os esforços no processo de descrição para criar novas possibilidades de navegação aos usuários. Por fim, algumas iniciativas são apresentadas com o propósito de criar sistemas que são capazes de ligar dados em ambientes informacionais distintos.

Inf. Inf., Londrina, v. 22, n. 2, p. 449 - 466, maio/ago., 2017. http:www.uel.br/revistas/informacao/ 
Ana Carolina Simionato, Felipe Augusto Arakaki, Plácida Leopoldina Ventura Amorim da Costa Santos

Descrição em bibliotecas, arquivos, museus e galerias de arte: linkando recursos e comunidades

\section{PRINCÍPIOS DA DESCRIÇÃO NA CIÊNCIA DA INFORMAÇÃO}

Cada campo de estudo (Biblioteconomia, Arquivologia e Museologia) apresentam distinções e especificidades em suas teorias e práticas relacionadas ao fazer profissional. Inicialmente o que as difere são os objetivos, no entanto, as tipologias dos recursos informacionais, o contato e a forma de relação com o público definem o contexto da instituição. Entretanto, independentemente de suas diferenças, as aproximações são pautadas pelas tradições históricas da Biblioteconomia, da Museologia e da Documentação, além dos elementos que equilibram os fazeres e da aplicação de saberes no tratamento e descrição dos recursos informacionais em múltiplas as áreas.

A representação para o usuário deve ser apresentada de uma forma clara e sucinta. Isso não quer dizer que a descrição dos catálogos e instrumentos de pesquisa serão simplificados, mas sim, que serão estruturados com a finalidade de acesso e localização, uso e reuso. Simionato (2015, p.69) afirma que os processos de representação no contexto da Ciência da Informação pressupõem que devem "[...] identificar as propriedades distintas que identifiquem o recurso informacional como único, além de proporcionar formas de acesso, como os catálogos a seus usuários." Da mesma forma, o conceito de descrição, segundo Glushko (2013, p.143, tradução nossa) é

[...] frequentemente utilizado em seu sentido comum para significar as características visíveis ou importantes que caracterizam ou representam alguma coisa. No entanto, por vezes o conceito é mais precisamente utilizado no contexto da organização de sistemas, onde a descrição de recursos muitas vezes é mais formal, sistemática e institucional.

A descrição de recursos informacionais independente do domínio é realizada pelas características designadas à tipologia do recurso. Em arquivos, os processos descritivos envolvem correntes teóricas que desencadeiam a maneira de gerenciar os documentos. Entre elas, a Arquivística Integrada proposta por Rousseau e Couture (1998) oferece o tratamento informacional desde a fase corrente do arquivo, seguindo a fase intermediária e permanente, contemplando "[...] a construção de mecanismos eficientes para o acesso, os

Inf. Inf., Londrina, v. 22, n. 2, p. 449 - 466, maio/ago., 2017. 
Ana Carolina Simionato, Felipe Augusto Arakaki, Plácida Leopoldina Ventura Amorim da Costa Santos

Descrição em bibliotecas, arquivos, museus e galerias de arte: linkando recursos e comunidades

relacionamentos entre os próprios documentos e suas atividades de gerenciamento e controle do acervo em todas as suas idades." (SIMIONATO, 2015, p.83).

Yakel (2003) descreve que a função do arquivo é identificada pela descrição do arranjo e abrange a representação do próprio arquivo. Sendo a atribuição de valor dos metadados, realizada a partir da compreensão dos contextos administrativos e do ciclo das funções arquivísticas envolvendo gestão, identificação e localização dos documentos e disposta em instrumentos de pesquisa.

Em bibliotecas, o processo de descrição de recursos informacionais, denominado como catalogação, objetiva a construir as formas de representação para alimentação de catálogos a partir da descrição padronizada de recursos informacionais, contemplando sua forma, seu conteúdo e o seu arranjo em acervos (SANTOS, 2013).

O processo de catalogação além de garantir a unicidade ao recurso informacional de uma forma sucinta e estruturada dos dados, apresenta princípios de integridade das informações do registro, clareza à transmissão de informações ao usuário, precisão em um único conceito, organização lógica e consistência de informações semelhante (MEY; SILVEIRA, 2009).

Diante desse cenário, o modelo conceitual para estruturação de registros bibliográficos denominado de Functional Requirements for Bibliographic Records (FRBR - Requisitos Funcionais para Registros Bibliográficos) foi criado

[...] para o universo bibliográfico e para a compreensão de profissionais da área da Biblioteconomia e da Ciência da Informação, o que levou seus desenvolvedores a realizarem certas modificações na forma de apresentação dos conceitos pertinentes à construção de um modelo conceitual. (SILVA; SANTOS, 2013, p.124)

Isso influenciou o desenvolvimento de outros padrões como o BIBFRAME que tem a proposta de substituir o formato de intercâmbio Machine Readable Catalogin (MARC 21). Segundo Silva e Santos (2013) e Arakaki (2016) o BIBFRAME é caracterizado pela flexibilidade; possui uma arquitetura 
Ana Carolina Simionato, Felipe Augusto Arakaki, Plácida Leopoldina Ventura Amorim da Costa Santos

Descrição em bibliotecas, arquivos, museus e galerias de arte: linkando recursos e comunidades

para expressar e conectar informações; pode ser adotado além da comunidade de bibliotecas, entre outras características.

Os processos de descrição em galerias de arte são semelhantes aos processos nos museus e bibliotecas, no entanto, o enfoque é direcionado à comercialização de peças de arte. A descrição museológica é um processo muito semelhante à catalogação, os registros são criados para que conduzam a organização do catálogo, e estabeleça o controle para os aspectos organizacionais sobre todos os recursos informacionais da instituição (FERREZ, 1994).

Os instrumentos documentais devem ser sistematicamente seguidos e sempre atualizados, principalmente para que consiga ser realizado o acesso e localização do objeto. É necessário ter um corpo documental conciso, para que não haja repetições de números de registro. A decodificação, no âmbito da catalogação, "[...] liga-se diretamente à morfologia do objeto, isto é, diz respeito a materiais e técnicas de confecção, a formas, ornamentos, a partes constituintes, a funções utilitárias para as quais foi concebido e a significados simbólicos relacionados às formas materiais de representação." (BARBUY, 2002, p.71).

Na perspectiva de estabelecer uma estrutura que busca conciliar dados gerados em Museus foi desenvolvido o CIDOC-CRM. Carrasco, Thaller e Vidotti (2015, p.211) explicam que o "Cidoc Conceptual Reference Model (CIDOC CRM) é uma ontologia do domínio do patrimônio cultural, criada como uma ferramenta para integração da informação." Santos (2016, p.60) complementa que o CIDOC-CRM “[...] é uma ontologia formal de referência que busca atender a uma crescente demanda por pesquisa orientada, estudos comparativos, transferência de dados e migração de dados entre fontes heterogêneas de conteúdos culturais."

Em paralelo, têm-se apresentadas outras possibilidades de acesso de recursos a partir do mobile. Emaldi et al. (2012) apresentaram uma arquitetura para unificar Quick Response Code - QR Codes que liga informações no 
Ana Carolina Simionato, Felipe Augusto Arakaki, Plácida Leopoldina Ventura Amorim da Costa Santos

Descrição em bibliotecas, arquivos, museus e galerias de arte: linkando recursos e comunidades

contexto de patrimônios culturais. Dessa forma, o usuário captura o QR Code e o aplicativo retorna informações e áudio sobre o recurso buscado.

Outra possibilidade de uso de mobile foi relatado por Oomen, Baltussen e Van Erp (2012) alguns aplicativos que possibilitam a identificação do local do usuário a partir da captura de uma imagem, como por exemplo, Vistory ${ }^{1}$, Agora $^{2}$, e o Mobile City Guide. Nesse contexto, Alves et al. (2015) discutem a importância da representação para descrição de materiais de viagem como parte da preservação da memória pessoal e familiar.

O desenvolvimento de sistemas de gerenciamento tem evoluído a partir das tecnologias de informação. Essas mudanças ocorreram gradualmente e apresentaram reflexos nos procedimentos do profissional da informação que deve acompanhar esse desenvolvimento. Nesse sentido, é exigido algumas competências e entendimento mínimo de práticas da computação como linguagens de marcação como a eXtensible Markup Language (XML), da arquitetura Resource Description Framework (RDF) no auxílio da construção de representações a partir dos metadados e curadoria digital entre outros temas.

O conhecimento dessas ferramentas é necessário também para auxiliar no desenvolvimento e construção de padrões de metadados e mapeamento de padrões heterogêneos, ou também conhecidos como harmonização. Marcondes (2016) aponta os desafios no processo de harmonização entre as instituições, que complementado por Carrasco, Thaller e Vidotti (2015, p.211) os desafios permeiam estruturas, como a dos "[...] modelos de dados são projetados a partir exigências de cada comunidade, nem sempre considerando os requisitos de interoperabilidade entre elas."

A interoperabilidade é importante para gestão dos ambientes heterogêneos, mas para que um sistema consiga realizá-la, são necessários de um conjunto de ferramentas, tecnologias, estruturas, entre outros aspectos.

${ }^{1}$ Site: http://www.vistory.nl/

2 Site: http://agora.cs.vu.nl

Inf. Inf., Londrina, v. 22, n. 2, p. 449 - 466, maio/ago., 2017. http:www.uel.br/revistas/informacao/ 
Ana Carolina Simionato, Felipe Augusto Arakaki, Plácida Leopoldina Ventura Amorim da Costa Santos

Descrição em bibliotecas, arquivos, museus e galerias de arte: linkando recursos e comunidades

Enquanto objetos em bibliotecas e museus são individuais - 0 documento, a peça museológica -, os arquivos têm seu foco em conjuntos de documentos, os fundos. Mais complicado ainda é superar o isolamento dos sistemas de arquivos, bibliotecas e museus e integrá-los a outros sistemas existentes na Web, como enciclopédias, dicionários, bases de dados factuais, científicas e estatísticas, sistemas de georeferenciamento como o Geonames, bancos de imagens, de vídeos etc. (MARCONDES, 2012, p.181).

A interoperabilidade envolve-se diretamente no processo de escolha dos metadados que irão compor a descrição dos recursos em um sistema de informação. Santos, Simionato e Arakaki (2013) apresentaram a Metodologia BEAM como diretriz para profissionais escolherem e definirem os metadados que irão compor a descrição de recursos informacionais. Apesar dos autores focarem na escolha dos campos do formato MARC 21, pode ser aplicável em qualquer outro padrão de metadados para auxiliar na identificação de quais metadados são importantes e quais não serão utilizados. Os autores utilizam como base, o método Crosswalk que segundo St.Pierre e LaPlant (1998) é a correspondência de um padrão de metadados para outro. É um método utilizado antes do processo de interoperabilidade entre os sistemas. "Crosswalks fornecem capacidade de fazer o conteúdo dos metadados definidos em um padrão de metadados disponíveis para as comunidades que utilizam padrões de metadados relacionados." (ST. PIERRE, LAPLANT, 1998, tradução nossa, não paginado).

Nesse contexto, para adequar as necessidades informacionais de determinadas instituições e minimizar os problemas de interoperabilidade, a Dublin Core Metadata Initiative (DCMI) propôs diretrizes para regularização e estruturação de documentos do conjunto de metadados denominado de Dublin Core Application Profiles (DCAP), em português, Perfil de Aplicação Dublin Core. O DCAP é definido por Coyle e Baker (2009) como um documento (ou conjunto de documentos) que especifica e descreve os metadados utilizados para uma aplicação em um contexto particular.

O Dublin Core surgiu em 1995, em um Workshop realizado em Dublin, Ohio (EUA). Resultado desse evento, surgiu o Dublin Core Metadata Set, nome

Inf. Inf., Londrina, v. 22, n. 2, p. 449 - 466, maio/ago., 2017. http:www.uel.br/revistas/informacao/ 
Ana Carolina Simionato, Felipe Augusto Arakaki, Plácida Leopoldina Ventura Amorim da Costa Santos

Descrição em bibliotecas, arquivos, museus e galerias de arte: linkando recursos e comunidades

para referenciar os 15 elementos, sendo todos opcionais e repetíveis. Para aumentar o nível em granularidade da descrição a opção é utilizar o Dublin Core qualificado, que possui alguns elementos adicionais e qualificadores para especificar melhor determinados elementos como a data ou tipo de relação. Caso ainda seja necessário utilizar uma descrição mais detalhada de um recurso informacional é possível criar um Perfil de Aplicação e são frequentemente utilizados em Repositórios Digitais.

Basicamente o Perfil de Aplicação Dublin Core apresenta alguns passos para sua construção, como a definição dos Requisitos Funcionais que ele poderá ou não realizar com a descrição, a estruturação do Modelo de Domínio que estrutura quais recursos serão descritos e como eles se relacionam. Os aspectos que envolvem a descrição e os padrões de metadados têm-se apresentados relacionados com a Web Semântica e o uso das tecnologias do Linked Data para ligação de dados. Segundo Bizer; Heath; Berners-Lee (2009, não paginado, tradução nossa) explicaram que

\footnotetext{
Em resumo, Linked Data é simplesmente sobre como usar a Web para criar ligações entre os dados digitados a partir de diferentes fontes. [...] Tecnicamente, Linked Data refere-se a dados publicados na Web, de tal forma que é legível por máquina, o seu significado é explicitamente definido, ele está ligado a outros conjuntos de dados externos, e pode por sua vez ser ligados a partir de conjuntos de dados externos.
}

Exemplos de padrões que podem ser utilizados na proposta do Linked Data são o Schema.org, o BIBFRAME e o Dublin Core, entre outros. Segundo Wallis (2015) o Schema.org surgiu de uma iniciativa de buscadores como Google, Yahoo, Bing e Yandex, para desenvolver uma estrutura que seja capaz de melhorar a busca de informações. O Schema.org pode ser utilizado em diversos contextos como blogs e sites, entre outras plataformas que desejem estruturar suas informações no contexto da Web Semântica, para apresentar informações enriquecidas semanticamente. No contexto das bibliotecas, 0 Schema.org foi adotado como padrão para descrição de recursos do WorldCat (Catálogo da Online Computer Library Center - OCLC). O WorldCat possui mais 330 milhões de registros bibliográficos e indica ao usuário qual o recurso

Inf. Inf., Londrina, v. 22, n. 2, p. 449 - 466, maio/ago., 2017. http:www.uel.br/revistas/informacao/ 
Ana Carolina Simionato, Felipe Augusto Arakaki, Plácida Leopoldina Ventura Amorim da Costa Santos

Descrição em bibliotecas, arquivos, museus e galerias de arte: linkando recursos e comunidades

que está mais perto dele, além de apresentar e recomendar outros recursos (ARAKAKI, 2016).

Diante dessas perspectivas, bibliotecas, arquivos, museus e galerias de arte têm trabalhado na estruturação de seus catálogos a partir dessas tendências e possibilidade de otimizar a recuperação e navegação dos dados de acordo com as necessidades informacionais de seus usuários.

Com o crescimento de documentos disponibilizados no formato digital, há necessidade de evitar os esforços da representação dos recursos informacionais. Diante dessa perspectiva, um movimento envolvendo bibliotecas, arquivos e museus vêm discutindo as possibilidades das práticas da ligação de dados abertos (Linked Open Data - LOD) nesses ambientes denominado de Linked Open Data in Libraries, Archives and Museums (LODLAM).

Em 2011 ocorreu a primeira reunião em São Francisco,
Califórnia (EUA) e que tem se mantida a cada dois anos. A
segunda edição do evento ocorreu em Montreal em Quebec
(Canadá) e a terceira edição foi em Sidney na Austrália. O
intuito das reuniões foi: Identificar as ferramentas e técnicas
para a publicação e trabalhar com Linked Open Data. Os
projetos de precedentes e política de considerações de
licenciamento e direitos autorais sobre a publicação de
metadados biblioteca, arquivo e museu. [...] Publicar definições
e promover casos de uso que Ihe dão equipe LAM as
ferramentas que precisam para utilizar Linked Open Data em
suas instituições. (VOSS, 2012, não paginado, tradução
nossa).

No contexto brasileiro, esse cenário é representado pelo evento INTEGRAR e teve sua primeira edição no ano de 2002 com o tema "Integração Presente e Futura", e em 2006 com o tema "Compartilhando o conhecimento: o acesso sem fronteiras". Dez anos depois, houve a terceira edição em $2016 \mathrm{com}$ o tema "Preservar para as futuras gerações". O INTEGRAR foi concebido como um evento multidisciplinar, com o objetivo de discutir a integração dos atuantes em arquivos, bibliotecas, museus, centros de documentação e centros de memória.

Inf. Inf., Londrina, v. 22, n. 2, p. 449 - 466, maio/ago., 2017. http:www.uel.br/revistas/informacao/ 
Ana Carolina Simionato, Felipe Augusto Arakaki, Plácida Leopoldina Ventura Amorim da Costa Santos

Descrição em bibliotecas, arquivos, museus e galerias de arte: linkando recursos e comunidades

Os estudos sobre a integração e em especial utilizando a proposta do LOD, ampliam as possibilidades de acesso por meio dos relacionamentos propostos, criando uma gama de outras possibilidades com sistemas de descoberta mais sofisticados. Assim, o catálogo poderá remeter a outros recursos que estão fora da biblioteca direcionando para um museu, algum arquivo ou catálogo de uma galeria de arte.

Abrangendo o contexto das bibliotecas, dos arquivos, dos museus e das galerias de arte, surgiu ainda o movimento Linked Open Data Galleries, Libraries, Archives and Museum (LODGLAM). Com a diversidade de padrões e esquemas para descrição de recursos informacionais heterogêneos, observa que há uma tendência de diversas instituições a colaborarem na construção de estruturas que possam trocar informações.

As Galerias de Arte abrangem o viés comercial, no entanto, a representação é muito similar aos museus, podem-se aproveitar os dados utilizados no museu, ou até mesmo quando uma obra se encontra em exposição, pode-se direcionar ao catálogo das Galerias de Arte para indicar obras relacionadas e que a pessoa possa adquiri-las.

Um dos principais modelos que tem atingindo os contextos das Galerias, Bibliotecas, Arquivos e Museus é o caso da Europeana. A Europeana desenvolveu um modelo de dados (Europeana Data Model - EDM) que permite que instituições possam disponibilizar seus dados no contexto do Open Galleries, Libraries, Archives and Museum (OpenGLAM). O OpenGLAM é uma iniciativa Fundada pela European Commission e coordenada pela Open Knowledge.

O EDM busca padronizar descrições de recursos de patrimônio cultural de instituições como bibliotecas, arquivos, museus e galerias no modelo Linked Open Data. O modelo EDM tem como base, diretrizes e padrões internacionalmente reconhecidos como o Resource Description Framework (RDF) e Resource Description Framework Schema (RDFS), Web Ontology Language (OWL) para estruturação dos metadados, o padrão de metadados

Inf. Inf., Londrina, v. 22, n. 2, p. 449 - 466, maio/ago., 2017. http:www.uel.br/revistas/informacao/ 
Ana Carolina Simionato, Felipe Augusto Arakaki, Plácida Leopoldina Ventura Amorim da Costa Santos

Descrição em bibliotecas, arquivos, museus e galerias de arte: linkando recursos e comunidades

Dublin Core para descrição dos recursos, para representação dos vocabulários utiliza o Simple Knowledge Organization System (SKOS).

O modelo EDM utiliza ainda outras ontologias como Open Archives Initiative Object Resue \& Exchange (OAI-ORE) para organização de objetos e representações digitais, para descrição de autoridade de pessoas e instituições utiliza o Friend of a Friend (FOAF), além de especificações do próprio EDM. Outro exemplo dos esforços de cooperação entre bibliotecas arquivos e museus é a Digital Public Library America (DPLA). A DPLA criou um perfil de aplicação chamado de Metadata Application Profile (MAP) que utiliza como base o Dublin Core terms.

Desta forma, com o fluxo de informação cada vez mais intenso, os profissionais necessitam de ferramentas e saber que auxiliem 0 reaproveitamento de dados para assim evitar o retrabalho dos fazeres profissional e da representação dos recursos informacionais. Por conseguinte, o surgimento do Linked Data, reforçou as propostas de cooperação entre bibliotecas, arquivos, museus e ainda foram incluídas as galerias de arte que desempenham um papel fundamental na disseminação da informação e que também necessita de uma representação adequada para que seus recursos possam ser localizados.

\section{CONSIDERAÇÕES FINAIS}

Diante do uso das tecnologias que estão inseridas no fazer profissional, é necessário que o profissional adquira habilidades e competências para otimizar os processos de representação e construção de sistemas de gerenciamento de informação. Diversas são as frentes de atuação do profissional da informação, podendo atuar desde no auxílio da modelagem de banco de dados, dialogando com os profissionais do campo das engenharias e computação, como também, ampliar as possibilidades de acesso dos usuários aos recursos informacionais, uma vez que, os profissionais da informação conhecem seus usuários e suas necessidades informacionais.

Inf. Inf., Londrina, v. 22, n. 2, p. 449 - 466, maio/ago., 2017. http:www.uel.br/revistas/informacao/ 
Ana Carolina Simionato, Felipe Augusto Arakaki, Plácida Leopoldina Ventura Amorim da Costa Santos

Descrição em bibliotecas, arquivos, museus e galerias de arte: linkando recursos e comunidades

A clareza e domínio do uso dos metadados e dos padrões de metadados garantem a integridade dos dados e evita ambiguidade e representações descontextualizadas e incompletas. Nesse sentido, há ainda a vertente de aproveitamento de dados de outras instituições por meio da interoperabilidade de dados. Para isso, o profissional bibliotecário, arquivista e museólogo, devem conhecer tanto o padrão de metadados de origem quanto o que estão sendo utilizado por sua instituição.

A aplicação proposta do Linked Data amplia as possibilita navegação dos usuários. Com os dados ligados, o usuário pode encontrar um livro de uma biblioteca e desse livro pode encontrar os arquivos originais (documentos históricos, manuscritos, etc.) para construção do livro, uma pintura relacionada ao livro ou a época que o livro está relacionado, que por sua vez pode encontrar informações da última exposição da pintura, se está em um museu ou em uma galeria de arte. Ao visitar um museu, uma galeria de arte, ou uma biblioteca, ou simplesmente uma consulta online, o uso do mobile pode auxiliar na propagação dessas informações e pode facilitar o acesso imediato desses recursos digitalmente.

Por fim, considera-se que a integração de bibliotecas, museus, arquivos e galerias de arte são fundamentais para proporcionar um ambiente ligado e ampliar as possibilidades de navegação dos usuários. Nesse contexto, a descrição adequada e o mapeamento do metadados são essenciais para promover diversas relações entre recursos, possibilitando aos usuários informações mais completas referentes às temáticas de seus interesses.

\section{REFERÊNCIAS}

ALVES, R. C. V. et al. BEAM repository: a proposal for family and personal repository. In: International Conference on Dublin Core and Metadata Applications. Anais... 2015. p. 136-145. Disponível em: $<$ http://dcpapers.dublincore.org/pubs/article/view/3769>. Acesso em: 31 maio 2017. 
Ana Carolina Simionato, Felipe Augusto Arakaki, Plácida Leopoldina Ventura Amorim da Costa Santos

Descrição em bibliotecas, arquivos, museus e galerias de arte: linkando recursos e comunidades

ARAKAKI, F. A. Linked Data: ligação de dados bibliográficos. 2016. 144 f.

Dissertação (Mestrado em Ciência da Informação) - Universidade Estadual

Paulista 'Júlio de Mesquita Filho', Marília/SP, 2016. Disponível em:

<https://repositorio.unesp.br/handle/11449/147979>. Acesso em: 30 maio 2017.

BARBUY, H. Os museus e seus acervos: sistemas de documentação em desenvolvimento. In: CONGRESSO INTERNACIONAL DE ARQUIVOS, BIBLIOTECAS, CENTROS DE DOCUMENTAÇÃO E MUSEUS, 01., 2002, São Paulo. Anais do INTEGRAR: $1^{\circ}$ Congresso Internacional De Arquivos, Bibliotecas, Centros de Documentação e Museus. São Paulo: Imprensa Oficial do Estado de SP, 2002.

BIZER, C.; HEATH, T.; BERNERS-LEE, T. Linked Data-the story so far. In: Semantic services, interoperability and web applications: emerging concepts. Information Science Reference (an imprint of IGI Global), USA, p. 205-227, 2009.

CARRASCO, L.; THALLER, M.; VIDOTTI, S. A. B. G. Ontologia Cidoc CRM no contexto dos ambientes digitais de patrimônios culturais. Liinc em Revista, Rio de Janeiro, v. 11, n. 1, 2015. Disponível em:

<http://revista.ibict.br/liinc/article/view/3628>. Acesso em: 31 maio 2017.

COYLE, K.; BAKER, T. Guidelines for Dublin Core application profiles.

DCMI, 2009. Disponível em: <http://dublincore.org/documents/profileguidelines/>. Acesso em: 31 maio 2017.

EMALDI, M. et al. LinkedQR: improving tourism experience through Linked Data and QR codes. In: INTERNATIONAL CONFERENCE ON UBIQUITOUS COMPUTING AND AMBIENT INTELLIGENCE, 6., Vitoria-Gasteiz, Spain.

Anais... Springer, 2012. Disponível em: <https://link.springer.com/book/10.1007/978-3-642-35377-2\#page=389>. Acesso em: 31 maio 2017.

FERREZ, H. D. Documentação museológica: teoria para uma boa prática. Cadernos de Ensaio, [S.I.], n. 2, p. 64-74, 1994.

GLUSHKO, R. J. The discipline of organizing. Massachusetts, EUA: The MIT Press, 2013.

MARCONDES, C. H. Linked Data - dados interligados - e interoperabilidade entre arquivos, bibliotecas e museus na web. Encontros Bibli: revista eletrônica de biblioteconomia e ciência da informação, Florianópolis, v. 17, n. 34, 9 ago. 2012. Disponível em:

<http://www.redalyc.org/html/147/14723061012/>. Acesso em: 31 maio 2017.

Inf. Inf., Londrina, v. 22, n. 2, p. 449 - 466, maio/ago., 2017. http:www.uel.br/revistas/informacao/ 
Ana Carolina Simionato, Felipe Augusto Arakaki, Plácida Leopoldina Ventura Amorim da Costa Santos

Descrição em bibliotecas, arquivos, museus e galerias de arte: linkando recursos e comunidades

MARCONDES, C. H. Interoperabilidade entre acervos digitais de arquivos, bibliotecas e museus: potencialidades das tecnologias de dados abertos interligados. Perspectivas em Ciência da Informação, Belo Horizonte, v. 21, n. 2, p. 61-83, jun. 2016. Disponível em:

<http://portaldeperiodicos.eci.ufmg.br/index.php/pci/article/view/2735>. Acesso em: 31 maio 2017.

MEY, E. S. A.; SILVEIRA, N. C. Catalogação no plural. Brasília: Briquet de Lemos, 2009.

OOMEN, J.; BALTUSSEN, L.; VAN ERP, M. Sharing cultural heritage the linked open data way: why you should sign up? In: MUSEUMS AND THE WEB, 16. São Diego, EUA, 2012. Anais... Museus e Web LLC, 2012. Disponível em: $<$ https://www.narcis.nl/publication/RecordID/oai:publications.beeldengeluid.nl:3 9>. Acesso em: 31 maio 2017.

PETERSON, D. Forms of representation: an interdisciplinary theme for cognitive science. [S.I.] Intellect Books, 1996.

PIERRE, M. S.; LAPLANT, W. P. Issues in crosswalking content metadata standards. MD: NISO Press, 1998. Disponível em:

<http://www.niso.org/publications/white papers/crosswalk/>. Acesso em: 31 maio 2017.

ROUSSEAU, J.-Y.; COUTURE, C. Os fundamentos da disciplina arquivística. Lisboa: Publicações Dom Quixote Lisboa, 1998.

SANTOS, H. P. Modelo CIDOC CRM: interoperabilidade semântica de informações culturais. Brazilian Journal of Information Science, Marília, v.10, n. 1, 2016. Disponível em:

<http://www2.marilia.unesp.br/revistas/index.php/bjis/article/view/5060>.

Acesso em: 31 maio 2017.

SANTOS, P. L. V. A. DA C. Catalogação, formas de representação e construções mentais. Tendências da Pesquisa Brasileira em Ciência da Informação, [S.I.], v. 6, n. 1, p. 1-24, 2013. Disponível em:

$<$ http://inseer.ibict.br/ancib/index.php/tpbci/article/view/100>. Acesso em: 31 maio 2017.

SANTOS, P. L. V. A. DA C.; SANTANA, R. C. G. Dado e Granularidade na perspectiva da Informação e Tecnologia: uma interpretação pela Ciência da Informação. Ciência da Informação, [S.I.], v. 42, n. 2, p. 199-209, 2013. Disponível em: <http://revista.ibict.br/ciinf/article/view/1382>. Acesso em: 31 maio 2017. 
Ana Carolina Simionato, Felipe Augusto Arakaki, Plácida Leopoldina Ventura Amorim da Costa Santos

Descrição em bibliotecas, arquivos, museus e galerias de arte: linkando recursos e comunidades

SANTOS, P. L. V. A. DA C.; SIMIONATO, A. C.; ARAKAKI, F. A. Definição de metadados para recursos informacionais: apresentação da metodologia BEAM. Informação \& Informação, Londrina, v. 19, n. 1, p. 146-163, fev. 2014.

Disponível em:

<http://www.uel.br/revistas/uel/index.php/informacao/article/view/15251>.

Acesso em: 31 maio 2017.

SANTOS, P. L. V. A. DA C.; VIDOTTI, S. A. B. G. Perspectivismo e tecnologias de informação e comunicação: acréscimos à Ciência da Informação?

DataGramaZero: revista de Ciência da Informação, Rio de Janeiro, n. 3. v. 10, 2009.

SILVA, R. E. DA; SANTOS, P. L. V. A. C. Requisitos Funcionais para Registros Bibliográficos (FRBR): considerações sobre o modelo e sua implementabilidade. RBBD. Revista Brasileira de Biblioteconomia e Documentação, São Paulo, v. 8, n. 2, p. 116-129, fev. 2013. Disponível em: $<$ https://febab.emnuvens.com.br/rbbd/article/view/214>. Acesso em: 31 maio. 2017.

SIMIONATO, A. C. Modelagem conceitual DILAM: princípios descritivos de arquivos, bibliotecas e museus para o recurso imagético digital. 2015. $200 \mathrm{f}$. Tese (Doutorado em Ciência da Informação) - Universidade Estadual Paulista 'Júlio de Mesquita Filho', Marília/SP, 2015.

<https://repositorio.unesp.br/handle/11449/123318>. Acesso em: 31 maio. 2017.

VOSS, J. Radically open cultural heritage data. 2012. Disponível em: $<$ http://www.museumsandtheweb.com/mw2012/papers/radically open cultural heritage data on the $w>$. Acesso em: 31 maio. 2017.

WALLIS, R. Schema.org. 2015. Disponível em: <http://schema.org/docs/howwework.html>. Acesso em: 31 maio. 2017.

YAKEL, E. Archival representation. Archival Science, [S.I.] v. 3, n. 1, p. 1-25, 2003. Disponível em:

$<$ https://pdfs.semanticscholar.org/7dc9/ebd0657b0d343e4e39ff8851f9c6ebf946 f6.pdf>. Acesso em: 31 maio. 2017.

Title

Description in libraries, archives, museums and art galleries: linking resources and communities

Abstract:

Introduction: The Web platform and the technologies allow for different forms of interaction between libraries, archives, museums, art galleries and their respective

Inf. Inf., Londrina, v. 22, n. 2, p. 449 - 466, maio/ago., 2017.

http:www.uel.br/revistas/informacao/ 
Ana Carolina Simionato, Felipe Augusto Arakaki, Plácida Leopoldina Ventura Amorim da Costa Santos

Descrição em bibliotecas, arquivos, museus e galerias de arte: linkando recursos e comunidades

public. The constant need to update information on professional technological development, putting the issue of this study, which is to identify which tendencies to optimize the channels of communication between user and informational resources, expanding the possibilities of representation in archives, libraries, museums and art galleries? Objective: The goal is to present emerging issues in the field of information representation in the Web platform for information workers in the context of libraries, archives, museums and art galleries. Methodology: It is characterized as a theoretical study, because under the optical search discuss the information representation challenges for integration and cooperation of different contexts, unified by the descriptions of informational resources, thus enabling the user navigation of informational environments and the location of other informational resources and information. Results: In this context, the principles of representation were raised in libraries, archives, museums and art galleries, seeking to highlight its characteristics and perceptions of the needs of users. It was later discussed the trends and challenges for information workers to reduce the representation's efforts to create new opportunities for navigation users. Conclusions: Finally, it was submitted a few initiatives that aims to create systems that can bind data of informational environments. It is concluded that the proposal of the reuse of the data seems to be an alternative to minimize efforts of professional rework.

Keywords: Libraries, archives, museums and art galleries. Semantic Web. Linked Data. Description and cataloguing.

\section{Titulo}

Descripción en bibliotecas, archivos, museos y galerias de arte: linkando recursos y comunidades

\section{Resumen:}

Introducción: La plataforma Web y las tecnologías han posibilitado diferentes formas de interacción entre bibliotecas, archivos, museos, galerías de arte y sus respectivos públicos. La constante necesidad de actualización del profesional de la información ante el desarrollo tecnológico, contextualizan la problemática de este estudio, que consiste en identificar cuáles son las tendencias que permiten optimizar los canales de comunicación entre usuario y los recursos informacionales, ampliando las posibilidades de representación en archivos, bibliotecas, museos y galerías de arte. Objetivo: El objetivo es presentar las temáticas emergentes en el ámbito de la representación de información en la plataforma Web para profesionales de la información en el contexto de bibliotecas, archivos, museos y galerías de arte. Metodología: Se caracteriza como un estudio teórico, que busca discutir bajo la óptica de la representación de la información los desafíos para integración y cooperación de diferentes contextos, unificados por las descripciones de recursos informacionales, posibilitando así, la navegación del usuario en los ambientes informacionales y la localización de otras informaciones y recursos informacionales. Resultados: Ante este contexto, se levantaron los principios de representación en bibliotecas, archivos, museos y galerías de arte, buscando evidenciar sus características y percepciones de las necesidades de los usuarios. Posteriormente se discutieron las tendencias y desafíos para los profesionales de la información con el fin de reducir los esfuerzos de la representación para crear posibilidades de navegación a los usuarios. Conclusiones: Por último, se han presentado algunas iniciativas que tienen el propósito de crear sistemas capaces de conectar datos entre ambientes 
Ana Carolina Simionato, Felipe Augusto Arakaki, Plácida Leopoldina Ventura Amorim da Costa Santos

Descrição em bibliotecas, arquivos, museus e galerias de arte: linkando recursos e comunidades

informacionales distintos. Se concluye que la propuesta del reaprovechamiento de los datos parece ser una alternativa para minimizar esfuerzos en el retrabajo del profesional.

Palabras clave: Bibliotecas, archivos, museos y galerías de arte. Web Semántica. Linked Data. Descripción y catalogación.

Recebido: 30.08 .2017

Aceito: 30.09 .2017

Inf. Inf., Londrina, v. 22, n. 2, p. 449 - 466, maio/ago., 2017. 